\title{
CEREBRAL ABSCESS AS A COMPLICATION OF NEONATAL SEPSIS
}

\author{
BY \\ N. R. BUTLER, H. BARRIE and K. W. E. PAINE \\ From The Hospital for Sick Children, Great Ormond Street, London
}

(RECEIVED FOR PUBLICATION APRIL 16, 1957)

Our reason for reporting two cases of cerebral abscess secondary to neonatal sepsis is the apparent rarity with which this complication occurs when the relatively high incidence of infection in the newborn period is considered. Crosse and Macintosh (1953) found that $10 \%$ of babies delivered at home and $14 \%$ of those delivered in hospital developed infections in the first 10 days of life; yet only four examples of this complication appear to have been described so far. The only reported survival was described by Farley (1949) in a baby whose illness began with a small boil on the face. This was followed by thrombophlebitis, cavernous sinus thrombosis and eventually a very large abscess in the left frontal lobe. Johnson, Elmore and Adams (1953) reported three cases, all of which died and in all of which a maternal respiratory infection was thought to be the source of infection.

Holt (1898) reviewed 32 cases of cerebral abscess in children, five of whom were under the age of 5 months, but in none of these was the infection connected with neonatal sepsis. One of Sandford's two cases (1928) was probably an example, although he did not claim it as such. Since attention has focused on neonatal sepsis only relatively recently, it may well be that its association with the development of severe infections in later months has not been recognized in the past.

\section{Case Reports}

Case 1. Jacqueline E., an 8-week-old infant, was admitted in April, 1956, with failure to thrive. She had vomited intermittently from birth and her weight gain had averaged less than 2 oz. per week.

Both the pregnancy and labour were normal and she was born at home weighing $7 \mathrm{lb} .12 \mathrm{oz}$. There was difficulty in feeding her until the third day, when she vomited some reddish-black material. However, melaena was absent and breast feeding was soon satisfactorily established. Septic spots appeared on the left side of the face during the first few days and at the end of the first week; the left thumb became red and swollen; she became ill and lost $1 \mathrm{lb}$. in weight. The thumb was incised by the general practitioner and a course of intramuscular penicillin was prescribed. She appeared to make a good recovery but at the age of 3 weeks she again became acutely ill with vomiting, fever and weight loss, necessitating admission to a local hospital. There she was found to have an erysipeloid eruption of the left thigh which responded to further intramuscular penicillin. However, pyrexia recurred with looseness of the stools, and her general condition again deteriorated and she became dehydrated. Improvement began after a course of tetracycline, but the baby was taken home against medical advice. Her progress at home was not satisfactory; feeding was difficult and weight gain was exceedingly slow. Inevitably, experiments were made in varying the type and quantity of the feeds without benefit. She was admitted to The Hospital for Sick Children (under the care of Dr. Wilfrid Sheldon), as vomiting had recurred for the previous five days and her general condition was deteriorating. She had had no fits or focal neurological symptoms at any time before admission.

The father, mother and three siblings were healthy, but the mother had suffered from a febrile upper respiratory tract infection at the time of the present infant's delivery and a sibling developed acute tonsillitis a few days later.

On admission the child looked pale and ill; the temperature was $102^{\circ} \mathrm{F}$., the pulse 138 per minute and the respirations 30 per minute. The cry was vigorous and not high-pitched. The anterior fontanelle was tense, though not bulging, and the skull circumference was $14 \frac{1}{2}$ in. There was no nuchal rigidity, and the limbs were hypotonic. The retinal veins were full but there were no retinal haemorrhages and papilloedema was absent. There was no sign of any primary septic lesion. The liver and spleen were not enlarged and there were no skin haemorrhages.

A catheter specimen of urine was normal. The haemoglobin was $53 \%(7 \cdot 84 \mathrm{~g}$.) per $100 \mathrm{ml}$. and the total leucocytes 16,400 per c.mm. (neutrophils $73 \%$; lymphocytes $27 \%$ ). Electrophoresis of serum protein showed no deficiency of gamma globulin.

A subdural haematoma was suspected and bilateral subdural taps were therefore attempted on the day of admission. Whilst no fluid collection was encountered on the right side, the left side yielded $60 \mathrm{ml}$. of thick 


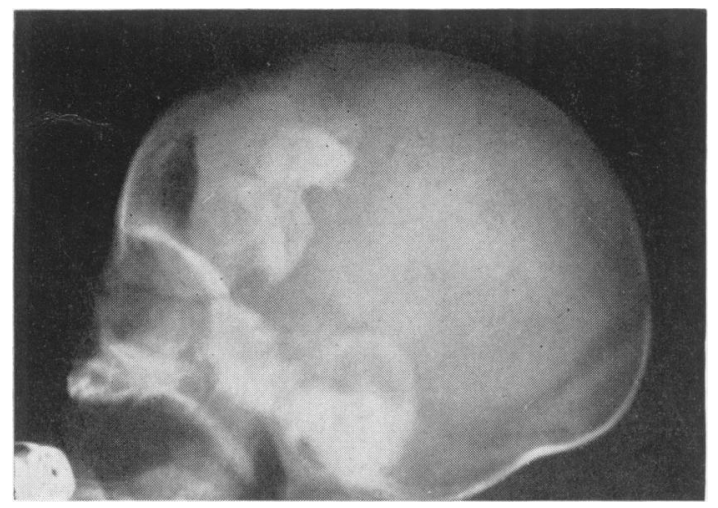

FIG. 1.-Skull plate of Case 1 showing the abscess cavity outlined by thorotrast and air.

yellow pus from which non-haemolytic streptococci were readily cultured. This organism was sensitive to all antibiotics. Intramuscular penicillin was given initially and was followed by a course of chloramphenicol for the first week. The following day a further $60 \mathrm{ml}$. of pus were aspirated, which again yielded the same organism. A blood transfusion was given which raised the haemoglobin level to $96 \%(14 \cdot 21 \mathrm{~g}$.) per $100 \mathrm{ml}$. Aspiration on the third day yielded $25 \mathrm{ml}$. of pus, now noticeably thinner. Plain radiographs of the skull showed only slight widening of the sutures. The baby was then transferred to the Neurosurgical Department under the care of Mr. Wylie McKissock. After radiographs following air replacement of the pus the extent of the cavity suggested that the abscess lay in the left frontal lobe rather than in the subdural space. This was confirmed the following day by outlining the cavity with thorotrast and air (Fig. 1).

Repeated aspiration was carried out with instillation of 10,000 units of crystalline pencillin and $50 \mathrm{mg}$. streptomycin. Gradually the quantity of pus diminished and it became less viscous. After 19 days' treatment no organisms could be seen in the stained smear and culture was sterile. The relatively long time needed to achieve this result was thought to be due to the formation of loculi in the abscess cavity, which led to the recovery of thin fluid or thick pus depending on which part of the cavity was aspirated. The temperature had remained normal since the fifth day and progress with regard to feeding had been equally satisfactory; the weight gain exceeded an average of $1 \mathrm{oz}$. per day. By the 27th day the abscess cavity had shrunk considerably (Fig. 2), but further aspiration produced no improvement. Therefore, on the 36th day, a left frontal lobectomy was carried out under general anaesthesia by one of us (K.W.E.P.). The frontal lobe was firm due to gliosis and, after removal, section of the frontal lobe showed that the specimen consisted of abscess cavity surrounded by gliosed brain, very little normal brain tissue having been removed.

The immediate post-operative period was complicated by a series of focal fits involving the right hand on the evening after operation. The fits were eventually con- trolled by phenobarbitone and the only neurological sequel was a mild right hemiparesis, which three weeks later was barely noticeable. Pyrexia of 100 to $101^{\circ} \mathrm{F}$., associated with collapse of the left lower lobe of the lung, occurred on the evening of the second post-operative day and resolved on the fifth day. Progress was thereafter uneventful; the baby remained afebrile and took feeds without vomiting.

On discharge, three weeks after operation, her general condition was excellent, the frontal wound was healed and the fontanelle was soft. A minimal right hemiparesis was still present.

She was re-admitted two weeks later as the mother had noticed that the fontanelle was bulging for the previous 24 hours. Radiography after aspiration of fluid from the left frontal cavity and air replacement revealed a subdural collection of fluid on the left side covering the whole hemisphere and communicating with the ventricular system by an opening in the left frontal horn. On July 25 a left parietal craniotomy was performed when the fluid was evacuated and the subdural membranes removed. The subdural space was obliterated by allowing the dura to collapse from the skull on to the underlying hemisphere. The child's immediate recovery was satisfactory but she died suddenly that same evening. A necropsy failed to reveal the immediate cause of death nor did it reveal any obstruction of the C.S.F. pathways as a cause of the subdural collection of fluid.

Case 2. John L. was admitted on May 21, 1953, at the age of 19 days having had convulsions for two days.

The pregnancy and labour were both normal, and the baby's birth weight was $8 \mathrm{lb}$. $2 \mathrm{oz}$. The condition was satisfactory until the fourth day when a boil appeared on the right side of the head. This was incised releasing pus and gradually disappeared, but by the 17 th day a swelling was noticed over the skull in the right posterior parietal region. The following day convulsive movements began in the left leg and became generalized. Lumbar puncture at a local hospital yielded cloudy cerebrospinal fluid under normal pressure. This con-

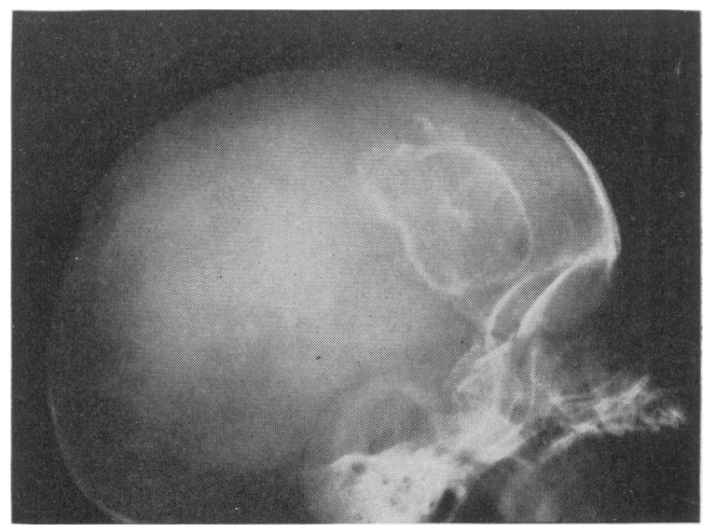

FIG. 2.-Skull plate of Case 1 showing the extent of the abscess cavity after four weeks' treatment. 
tained 1,400 leucocytes per c.mm. (neutrophils 52\%, monocytes $48 \%$ ), $180 \mathrm{mg}$. protein per $100 \mathrm{ml}$. and $45 \mathrm{mg}$. sugar per $100 \mathrm{ml}$. with no organisms on stained film or culture.

On transfer to the Neurosurgical Department at The Hospital for Sick Children, Great Ormond Street, his

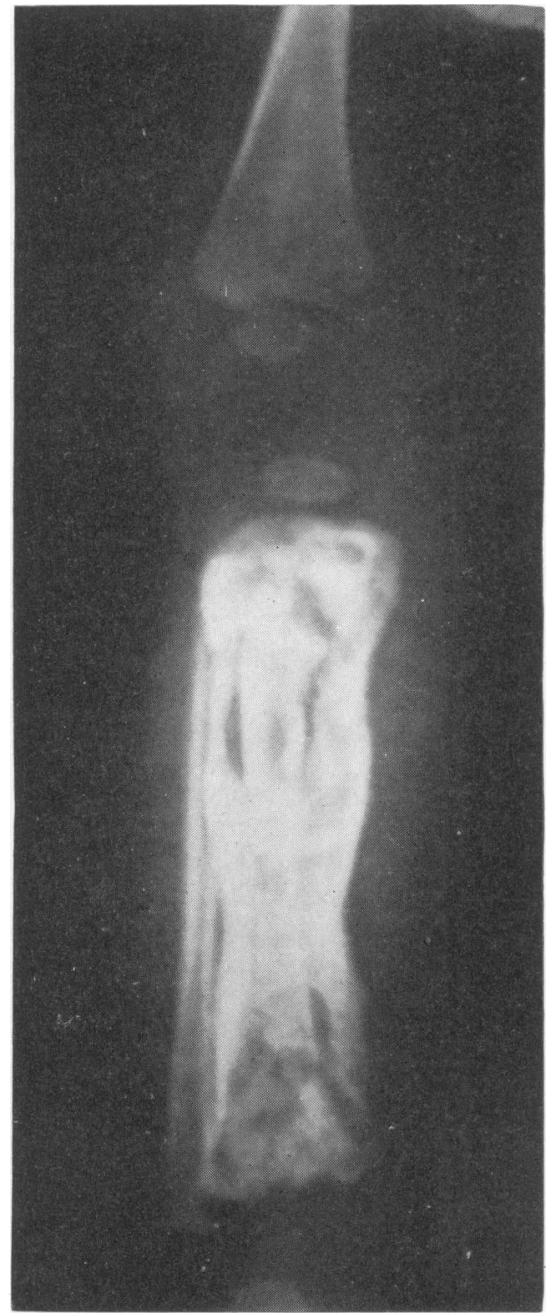

FIG. 3.-Radiograph of tibia of Case 2, showing osteomyelitis.

general condition was good. The anterior fontanelle tension was normal and there was no head retraction. A small scab marked the site of the healed pustule in the right parietal region where a soft fluctuant swelling, $3 \mathrm{~cm}$. in diameter, was palpable. The overlying skin was normal but the bone at the periphery of the swelling appeared to be elevated. Spontaneous movements were diminished in the left arm and leg, which were hypertonic with increased tendon reflexes.
Radiographs of the skull showed periosteal elevation with underlying osteoporosis at the site of the swelling. Ventriculography performed on the day of admission showed slight displacement of the third ventricle to the left and depression of the roof of the posterior part of the right lateral ventricle, the appearances being con-
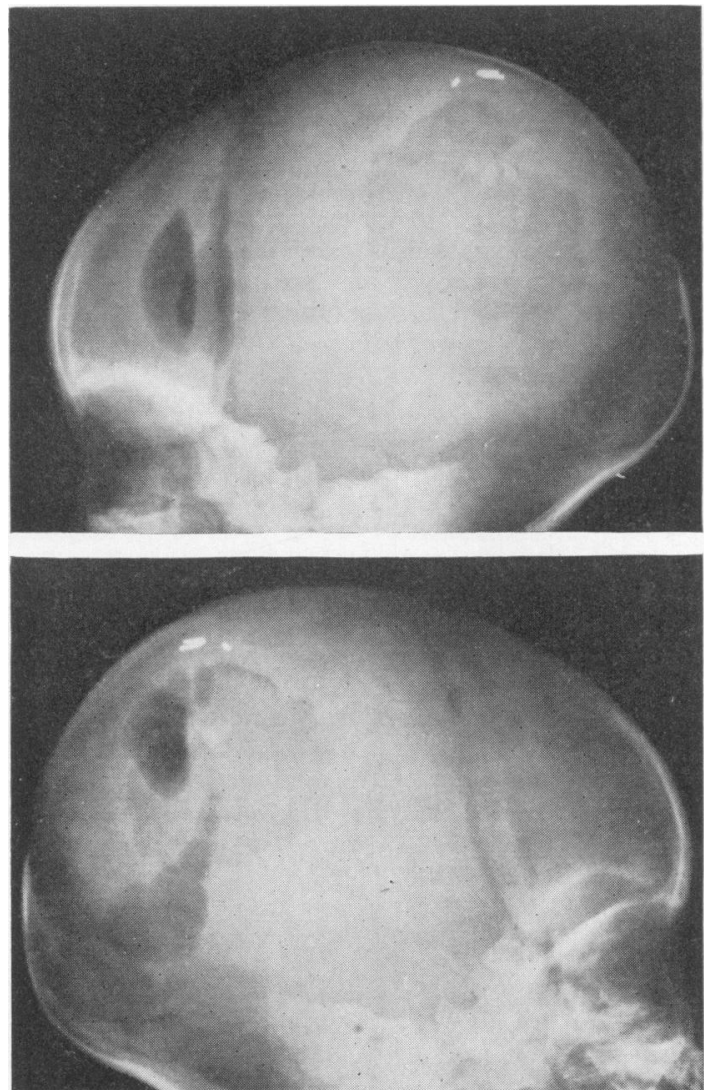

Fig. 4--Ventriculograms of Case 2 showing a large cavity in the right parietal region, dilatation of the anterior horns of the lateral ventricles, and the intercommunication. A large area of osteomyelitis of the skull can also be seen.

sistent with a right tempero-parietal space-occupying lesion. Craniotomy was performed on the same day when thick pus was released from a sub-periosteal abscess; removal of the infected part of the calvarium revealed an underlying extradural abscess with an intracerebral extension. Catheters were inserted into the extradural space and four-hourly instillations of penicillin begun.

The organism isolated from the pus proved to be a coagulase-positive staphylococcus, sensitive to penicillin and streptomycin both of which were given intramuscularly in large doses. The baby's general condition improved and the frequency of the convulsions diminished. On the 28th day of life, however, an area 
of cellulitis appeared in the left leg where intravenous fluids had been given, and a week later an abscess had localized, from which a coagulase-positive staphylococcus was again isolated. The dosage of penicillin was increased to 1 million units four-hourly and the streptomycin was changed to $0.25 \mathrm{~g}$. four-hourly of sulphafurazole to which the organism was also sensitive in vitro. However, this infection subsequently progressed to frank osteomyelitis of the tibia (Fig. 3).

The scalp wound healed satisfactorily, but a radiograph of the skull taken on the 34th day of life showed extensive bone erosion characteristic of osteomyelitis. A second operation was performed five days later when the affected bone was rongeured away and sequestra were removed. Daily lumbar punctures were done to reduce brain herniation, and, although the cerebrospinal fluid was sterile, it still contained an excess of leucocytes and protein. For the next three weeks the infant vomited at intervals and did not gain weight. The left arm and leg remained spastic.

On the 64th day of life, the convulsions began again and ventriculography was repeated. Symmetrical dilatation of the anterior horns of the lateral ventricles was demonstrated and air was shown to pass into a cavity in the right parietal region which communicated freely with the right lateral ventricle (Fig. 4). The baby's general condition gradually deteriorated after this; staphylococci appeared in the ventricular fluid and spinal fluid, and he died on August 4, 1953, aged 13 weeks and 5 days.

Dr. Martin Bodian at necropsy confirmed the presence of a large cerebral abscess in the right parietal lobe which had ruptured into the ventricle. There was also osteomyelitis of the skull and the left tibia. Toxic changes were present in the liver, heart and kidneys, as was a terminal pulmonary oedema.

\section{Discussion}

Spread of infection to the brain follows wellrecognized routes. Thus Case 2 is an example of direct spread from local osteomyelitis. From septic lesions of the face the infection may reach the brain via the emissary veins and the cavernous sinus (Farley, 1949). A blood-borne infection may also be responsible, recorded primary lesions including paronychia, pneumonia and umbilical sepsis. Agammaglobulinaemia is a rare cause of widespread neonatal infection, but the gamma globulin estimation done in our Case 1 was normal. An interesting association is the occurrence of cerebral abscess after splenectomy (Johnson et al., 1953). Although this was ascribed to infection of an intracerebral haematoma due to thrombocytopenic purpura, an increased liability to infection has been thought to follow splenectomy (Gofstein and Gellis, 1956). The susceptibility of the newborn is such as to render them liable to infection by organisms commonly regarded as non-pathogenic. Johnson et al. (1953), who stressed the danger of maternal infection at the time of birth, advocated immediate prophylactic treatment with antibiotics. Although the mother of Case 1 was not treated with an antibiotic, the infant was given intramuscular penicillin relatively early for a minor infection. Moreover, the responsible organism was a non-haemolytic streptococcus which, even if it had been isolated from the mother, would not have excited attention.

To add to the obvious difficulty of neurological localization at this age, the classical signs of raised intracranial pressure may be minimal or absent. Even if present, the causes of vomiting in infancy are protean and convulsions may be the non-specific response to fever of any cause. In neither of our cases was the heart rate slowed. Although any of the commonly described neurological manifestations of cerebral abscess can be present the only definite sign that may be encountered in infancy is a full anterior fontanelle. This raises the question of differential diagnosis from meningitis and other space-occupying lesions, particularly subdural haematoma, both of which are the more common conditions, and we admit that the clinical diagnosis may be extremely difficult. Subdural haematoma, which not infrequently produces a febrile systemic response, can only be exluded by a subdural tap. Meningitis usually gives rise to a more acute clinical picture with meningeal irritation, although the latter may be misleadingly absent. In our limited experience, we feel that cerebral abscess should be considered a possible diagnosis in any infant with a full anterior fontanelle, who has become gradually but increasingly ill following infection elsewhere in the body.

Once a cerebral abscess is suspected, localization may be possible on careful clinical examination; the abscess should then be aspirated and its extent determined radiologically after replacement with air or radio-opaque medium. Frequent aspiration and instillation of the appropriate antibiotic should be continued until the cavity begins to obliterate. In the case described by Farley (1949) no other operative measure was required and the cavity closed spontaneously after six months. In our Case 1, the cavity could not be reduced to less than $2 \mathrm{~cm}$. in size and for this reason, excision, amounting to a frontal lobectomy, was necessary.

When no evidence of localization is forthcoming, little further help can be expected from plain radiographs of the skull unless gas-forming organisms are present. Arteriography is the theoretical investigation of choice but is technically impracticable at this age. Similarly, the limitations of electro-encephalography in infancy detract from its 
usefulness in the localization of a suspected abscess. Lumbar puncture is often performed to exclude meningitis but is not without danger from prolapse of the cerebellum into the foramen magnum, although the flexibility of the sutures will preclude coning of the supratentorial structures. Ventriculography carries the risk of intraventricular rupture of the abscess, but this investigation, preceded by exploration of the subdural space, may be essential for localization. The abscess should then be aspirated immediately after ventriculography.

With regard to the prognosis, Farley's patient is the only one among those we have reviewed to have survived, and was well when demonstrated at the Royal Society of Medicine at the age of 6 years. Convulsions occurred during the first year and speech was retarded until the age of 4 , but the eventual mental development was normal (Farley, 1952). Subsequently, this child's fits recurred and a slight hemiparesis became detectable (Farley, 1957).

Our first patient, who had made a good recovery after a frontal lobectomy, died suddenly after a second operation for an accumulation of subdural fluid. This unusual complication should not be a contra-indication to excision of a residual abscess as the final stage of treatment in similar cases.

\section{Summary}

Two cases of cerebral abscess complicating neonatal sepsis are described. In one of these, a maternal infection was present at the time of birth. The diagnosis and treatment of the condition is discussed and the literature of cerebral abscess in the neonatal period is briefly reviewed.

We are indebted to Dr. Wilfred Sheldon and Mr. Wylie McKissock for permission to publish these cases, to Dr. Martin Bodian for the post-mortem findings in Case 2 and to Mr. Derek Martin for the illustrations.

\section{REFERENCES}

Crosse, V. M. and Mackintosh, J. H. (1953). Brit. med. J., 1, 1374. Farley, D. L. B. (1949). Lancet, 1, 264.

- (1952). Proc. roy. Soc. Med., 45, 400.

- (1957). Personal communication.

Gofstein, R. and Gellis, S. S. (1956). A.M.A. J. Dis. Child., 91, 566.

Holt, L. E. (1898). Arch. Pediat., 15, 81.

Johnson, G. D., Elmore, S. E. and Adams, F. F. (1953). J.S.C. med. Ass., 49, 281.

Sanford, H. N. (1928). Amer. J. Dis. Child., 35, 256 